Produto \& Produção, vol. 11, n. 1, p. 70 - 79, fev. 2010

Edição Metrologia

\title{
Determinação da Incerteza de Medição na Calibração da Área de Aberturas Circulares
}

Pedro B. Costa

Wellington S. Barros

Instituto Nacional de Metrologia Normalização e Qualidade Industrial - Inmetro

pbcosta@inmetro.gov.br

\section{RESUMO}

O Laboratório de Metrologia Dimensional do Inmetro está implementando um sistema para a medição de áreas de aberturas circulares utilizadas em medidas radiométricas e fotométricas. As medições são realizadas através da determinação das coordenadas dos pontos ao longo da borda da abertura. Dessa forma, é possível determinar o diâmetro médio, e, consequentemente, a área da abertura. Neste trabalho será descrito a metodologia para o calculo da incerteza de medição associada a este tipo de metodologia.

Palavras chave: Incerteza de medição, Abertura circular, Medição sem contato.

\section{INTRODUÇÃO}

Orifícios de formato e dimensões bem definidos, também denominados aberturas, são utilizados para a realização de medidas radiométricas e fotométricas. Assim, a calibração de áreas de aberturas é atualmente um ponto de grande importância para garantir a exatidão destas medições [1,2]. A divisão de metrologia óptica do Inmetro está atualmente desenvolvendo um projeto para a realização da grandeza de intensidade luminosa, onde é de grande importância à determinação da área de aberturas circulares utilizadas para essa padronização [3].

Acompanhando a tendência dos demais institutos nacionais de metrologia na medição de áreas de aberturas, o Laboratório de Metrologia Dimensional do Inmetro desenvolveu um sistema para medição desse tipo de padrão através de um método sem contato, diferentemente dos métodos tradicionais que utilizam Máquinas de Medir por Coordenadas (MMC's) ou métodos ópticos com a determinação da área através de ajustes de mínimos quadrados [4]. 


\section{DESCRIÇÃO DO SISTEMA}

O sistema desenvolvido para a medição de aberturas circulares é composto por um microscópio com uma mesa de deslocamentos horizontais (XY) e uma câmera CCD. A imagem ampliada da abertura é capturada pela câmera e enviada a um microcomputador. A seguir, são determinadas as coordenadas $X$ e $Y$ dos pontos referentes às bordas da abertura, a partir das quais se obtém o diâmetro da abertura e, consequentemente, sua área. A determinação destas coordenadas da borda é realizada automaticamente através de um software desenvolvido pelo próprio laboratório com um algoritmo específico para a detecção de bordas.

\subsection{Software de Medição}

A partir da imagem capturada pela câmera CCD, o software "Circular Aperture" realiza uma varredura nas direções $x$ e y da imagem para determinar as coordenadas, em valores de pixel, dos pontos da borda ao longo da abertura. $\mathrm{O}$ algoritmo utilizado para a determinação dessa borda analisa os valores correspondentes à intensidade luminosa de cada pixel ao longo de uma linha. A partir destes valores é possível modelar uma curva através de uma função $f(x)$. Os resultados do cálculo dos pontos de inflexão dessa função representam os pontos em que ocorre a transição claro-escuro na imagem, ou seja, representam os pontos das bordas da abertura.

O modelo matemático desenvolvido para a obtenção da área da abertura tem como primeiro passo a determinação do centro da circunferência. $O$ valor que representa o centro da circunferência no eixo $X$ é encontrado através do cálculo da média de todas as coordenadas medidas nesta direção. $O$ mesmo procedimento é realizado para a determinação do centro no eixo $\mathrm{Y}$.

Uma vez definido o centro da abertura, calcula-se a dimensão das hipotenusas dos triângulos retângulos definidos a partir das coordenadas dos pontos da borda e das coordenadas do centro, conforme mostrado na figura 1. Estas hipotenusas, por sua vez, representam os raios da circunferência em cada ponto de medição $i$.

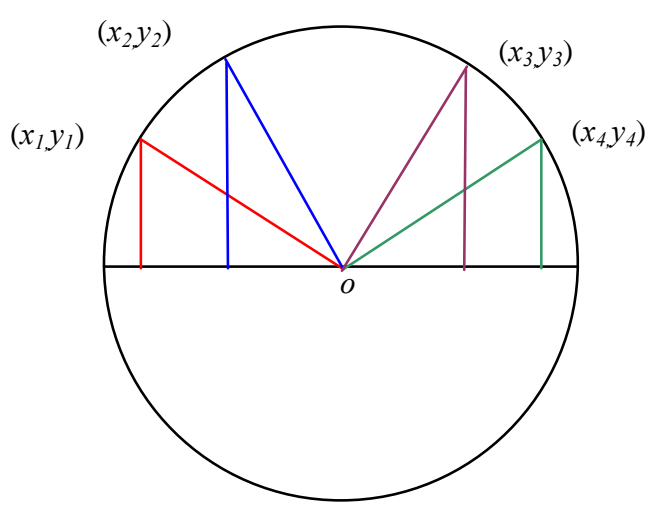

Figura. 1. Determinação dos raios através da construção de triângulos 
A área da abertura pode ser calculada em função de um raio médio obtido a partir dos diversos raios calculados. Dessa forma, obtém-se uma expressão para a área de abertura em função das coordenadas $x$ e y dos pontos localizados na borda da abertura (Eq. 1).

$$
A=\frac{\pi}{N} \cdot \sum_{i}^{N}\left[\left(x_{i}-C x\right)^{2}+\left(y_{i}-C y\right)^{2}\right]
$$

Para que o algoritmo elaborado seja capaz de identificar os pontos da borda da abertura e obter o valor das coordenadas destes pontos, é necessário que, com uma determinada ampliação do microscópio, toda a área da abertura esteja contida na imagem exibida na tela. Esta exigência associada ao algoritmo e ao microscópio utilizado para a medição restringe a utilização da metodologia atual desenvolvida à medição de aberturas de até $4 \mathrm{~mm}$ de diâmetro.

\section{INCERTEZA DE MEDIÇÃO}

O modelo matemático utilizado para obtenção do resultado final e avaliação da incerteza de medição deriva da Eq. 1. Entretanto, os resultados obtidos por meio da equação citada são valores contados em pixel. Para que seja possível obter valores em unidades de área, é necessário estabelecer a dimensão de um pixel, ou seja, definir quanto cada pixel visualizado na tela vale em termos de uma dada unidade de comprimento. Essa definição do tamanho de um pixel na direção x e na direção y foi obtida utilizando-se um laser interferométrico e uma escala graduada, usada apenas para obter uma imagem na tela.

O procedimento para a determinação dos valores de tamanho do pixel nas direções $x$ e y são inseridos no modelo de medição de aberturas como um padrão de referencia com valor médio e incerteza conhecidos.

O modelo matemático final para a calibração da área da abertura com os valores convertidos em unidade de comprimento é apresentado na Eq. 2. Neste modelo, considera-se também o efeito dilatação térmica sobre a superfície medida.

$$
A_{(A b e r t u r a)}=\frac{\left.\pi \cdot\left\{\left(x_{i}-C x\right) \cdot L p x\right]^{2}+\left[\left(y_{i}-C y\right) \cdot L p y\right]^{2}\right\}}{1+\beta \cdot \Delta t}
$$

Onde,

$x_{i} \quad$ Coordenadas da borda na direção $x$ em pixels

$C x \quad$ Centro da circunferência no eixo $x$ em pixels

$L p x \quad$ Comprimento do pixel no eixo $x$

$y_{i} \quad$ Coordenadas da borda na direção y em pixels

Cy Centro da circunferência no eixo y em pixels

Lpy Comprimento do pixel no eixo y

$\beta \quad$ Coeficiente de dilatação superficial da abertura

$\Delta t$ Diferença da temperatura da abertura em relação à temperatura de referência 
A determinação dos valores de Cx e Cy são realizadas através da média dos valores de todos os pontos ao redor da abertura, encontrado pelo programa de medição. (Eq. 3 e Eq. 4)

$$
\begin{aligned}
& C x=\frac{1}{n} \sum_{i=1}^{n} x_{i} \\
& C y=\frac{1}{n} \sum_{i=1}^{n} y_{i}
\end{aligned}
$$

\subsection{Incerteza do Tipo A}

No procedimento proposto para a medição, a abertura foi medida em cinco posições distintas. No total, foram medidos 3850 pontos. A partir de cada um dos pontos calcula-se um valor de área da abertura. Assim, a repetitividade foi avaliada a partir do desvio padrão destes valores de área. A componente de incerteza relacionada à repetitividade é relativamente pequena em comparação às demais, uma vez que a quantidade de medições é bastante elevada.

Os valores apresentados como exemplo, foram retirados da medição de uma abertura circular de $0,24 \mathrm{~mm}$. O valor médio medido da abertura foi de 0,04620 $\mathrm{mm} 2$, e o desvio padrão das medições igual a $0,00198 \mathrm{~mm} 2$. A repetitividade foi determinada de acordo com a equação 5.

$$
u(A)=\frac{s}{\sqrt{n}}=\frac{0,00198}{\sqrt{3850}}
$$

(5)

Obtendo o valor de 0,03 $\mathrm{m}^{2}$ como contribuição da repetitividade para a incerteza de medição. 


\subsubsection{Incerteza das Coordenadas de X}

As incertezas relacionadas as bordas do eixo $\mathrm{x}$, determinadas pelo programa, são estimadas como sendo o maior erro possível que o programa pode cometer na determinação de um ponto na borda da abertura. Esse valor é estimado como sendo de metade de um pixel, pois se a borda da abertura não estiver exatamente sobre a intersecção de dois pixels, e sim, sobre o meio de um pixel, o programa automaticamente ira detectar a borda como se esta estivesse sobre uma das extremidades deste pixel. A este valor é atribuída uma distribuição retangular pois a probabilidade da borda da abertura estar contida em um determinado ponto no interior de um pixel é a mesma para todos os pontos dentro deste pixel. 0 coeficiente de sensibilidade é calculado através da equação 6 .

$$
\frac{\partial A}{\partial x_{i}}=2 \pi \cdot\left(x_{i}-C x\right) \cdot \frac{L p x^{2}}{1+\beta \cdot \Delta t}
$$

\subsubsection{Incerteza das Coordenadas de y}

A incerteza devido ao erro na determinação das coordenadas de y é calculada de forma similar a incerteza das coordenadas de $\mathrm{x}$, variando apenas $\mathrm{O}$ cálculo do coeficiente de sensibilidade. (Eq 7).

$$
\frac{\partial A}{\partial y_{i}}=2 \pi \cdot\left(y_{i}-C y\right) \cdot \frac{L p y^{2}}{1+\beta \cdot \Delta t}
$$

\subsubsection{Incerteza da Determinação do Centro da Circunferência}

Para a determinação do centro da circunferência nos eixos $x$ e $y$, são utilizados os valores das bordas em ambos os eixos, o centro é determinado através da média desses valores. Portanto, o centro da circunferência é a média de uma série de valores.

Como forma de estimar uma dúvida para a determinação do centro da circunferência, é calculada a amplitude de todos os possíveis valores de centro utilizados no calculo da média. Este procedimento é valido para ambos os eixos.

Para a medição da abertura, citada no item 3.1, a amplitude encontrada para os eixos $x$ e y foram de 5,0 e 4,5 respectivamente (Valores em unidade de pixel).

Os coeficientes de sensibilidade para ambos os eixo são calculados através das equações 8 e 9 . 


$$
\begin{aligned}
& \frac{\partial A}{\partial C x}=(-2 \pi) \cdot\left(x_{i}-C x\right) \cdot \frac{L p x^{2}}{1+\beta \cdot \Delta t} \\
& \frac{\partial A}{\partial C y}=(-2 \pi) \cdot\left(y_{i}-C y\right) \cdot \frac{L p y^{2}}{1+\beta \cdot \Delta t}
\end{aligned}
$$

\subsubsection{Incerteza do Comprimento do Pixel}

O comprimento do pixel nos eixos $x$ e y são valores obtidos de uma calibração previa da imagem. $O$ valor verdadeiro convencional para o pixel é fornecido como a média de $\mathrm{n}$ valores com uma incerteza expandida atribuída, graus de liberdade infinito e fator de abrangência igual a dois.

No sistema utilizado pelo Lamed os pixels possuem valores de $0,000921 \mathrm{~mm}$ e $0,000930 \mathrm{~mm}$, para os eixos $x$ e y respectivamente. Com incerteza de $20 \mathrm{~nm}$ para ambos os eixos.

O coeficiente de sensibilidade relativo ao comprimento do pixel é calculado de acordo com as equações 10 e 11, mostradas a seguir.

$$
\begin{aligned}
& \frac{\partial A}{\partial L p x}=2 \pi \cdot\left(x_{i}-C x\right)^{2} \cdot \frac{L p x}{1+\beta \cdot \Delta t} \\
& \frac{\partial A}{\partial L p y}=2 \pi \cdot\left(y_{i}-C y\right)^{2} \cdot \frac{L p y}{1+\beta \cdot \Delta t}
\end{aligned}
$$

\subsubsection{Incerteza Devido às Condições Ambientais}

Como forma de considerar as contribuições devido as condições ambientais foi inserido no modelo matemático da calibração a correção relativa a dilatação térmica da abertura.

Para o cálculo da incerteza de medição, são consideradas como grandezas de entrada a diferença entra a temperatura da abertura e a temperatura de referência $\left(20^{\circ} \mathrm{C}\right)$ e o coeficiente de dilatação superficial da abertura.

Para a estimativa da variação da temperatura $(t)$ são feitas duas considerações. A primeira considera a incerteza do certificado de calibração do termômetro, e a segunda é considerada como a máxima variação da temperatura observada durante a calibração, nomeada como "Oscilação no tempo".

A abertura utilizada neste trabalho possui o coeficiente de dilatação de aproximadamente 11,5E-6 (Aço), este coeficiente não possui um valor de incerteza. Como este valor corresponde ao coeficiente de dilatação linear do aço, multiplicamos este valor por dois para obter o coeficiente de dilatação superficial do padrão e a este valor atribuímos uma estimativa de $1 \mathrm{E}-5$ para o cálculo da incerteza de medição. A variabilidade deste valor é julgada confiável a $10 \%$, obtendo assim através da equação 12, cinqüenta graus de liberdade para esta estimativa. 
$v_{i}=\frac{1}{2} \cdot\left[\frac{\Delta u\left(x_{i}\right)}{u\left(x_{i}\right)}\right]^{-2}=\frac{1}{2} \cdot(0,10)^{-2}$

Os coeficientes de sensibilidade relativos ao coeficiente de dilatação e a diferença de temperatura são calculados através das equações 13 e 14 .

$$
\begin{aligned}
& \frac{\partial A}{\partial \beta}=(-\pi) \cdot \frac{\left(x_{i}-C x\right)^{2} \cdot L p x^{2}+\left(y_{i}-C y\right)^{2} \cdot L p y^{2}}{(1+\beta \cdot \Delta t)^{2}} \cdot \Delta t \\
& \frac{\partial A}{\partial \Delta t}=(-\pi) \cdot \frac{\left(x_{i}-C x\right)^{2} \cdot L p x^{2}+\left(y_{i}-C y\right)^{2} \cdot L p y^{2}}{(1+\beta \cdot \Delta t)^{2}} \cdot \beta
\end{aligned}
$$

\subsubsection{Incerteza Padrão Combinada}

A incerteza padrão combinada da medição da abertura circular de $0,24 \mathrm{~mm}$ foi calculada de acordo com a equação 15, e os valores inseridos na equação foram extraídos da tabela 1.

$$
\begin{aligned}
& u_{c}^{2}(A)=\left[\frac{\partial A}{\partial x_{i}}\right]^{2} \cdot u^{2}\left(x_{i}\right)+\left[\frac{\partial A}{\partial y_{i}}\right]^{2} \cdot u^{2}\left(y_{i}\right)+\left[\frac{\partial A}{\partial C x}\right]^{2} \cdot u^{2}(C x)+\left[\frac{\partial A}{\partial C y}\right]^{2} \cdot u^{2}\left(x_{i}\right)+\left[\frac{\partial A}{\partial L p x}\right]^{2} \cdot u^{2}(L p x) \\
& +\left[\frac{\partial A}{\partial L p y}\right]^{2} \cdot u^{2}(L p y)+\left[\frac{\partial A}{\partial \beta}\right]^{2} \cdot u^{2}(\beta)+\left[\frac{\partial A}{\partial \Delta t}\right]^{2} \cdot u^{2}(\Delta t)++2 \cdot\left[\begin{array}{l}
\left(\frac{\partial A}{\partial x_{i}} \cdot \frac{\partial A}{\partial C x} \cdot u\left(x_{i}\right) \cdot u(C x) \cdot r\left(x_{i}, C x\right)\right)+ \\
\left(\frac{\partial A}{\partial y_{i}} \cdot \frac{\partial A}{\partial C y} \cdot u\left(y_{i}\right) \cdot u(C y) \cdot r\left(y_{i}, C y\right)\right)
\end{array}\right]
\end{aligned}
$$

\begin{tabular}{|c|c|c|c|c|c|}
\hline $\begin{array}{c}\text { Grandeza } \\
\text { de } \\
\text { influência }\end{array}$ & Distribuição & $\begin{array}{c}\text { u(xi) / } \\
\text { Unidade }\end{array}$ & $\mathrm{i}$ & Ci mm²/Unidade & $u(A) m^{2}$ \\
\hline$u(x)$ & $\mathrm{R}$ & 0,289 & Infinito & $7 \mathrm{E}-4 \cdot \mathrm{mm}^{2}$ & 0.0002 \\
\hline$u(y)$ & $\mathrm{R}$ & 0,289 & Infinito & $7 \mathrm{E}-4 \cdot \mathrm{mm}^{2}$ & 0.0002 \\
\hline$u(C x)$ & $\mathrm{N}$ & 2,5 & 169 & $-6.9 \mathrm{E}-4 \cdot \mathrm{mm}^{2}$ & 0.00173 \\
\hline $\mathrm{u}(\mathrm{Cy})$ & $\mathrm{N}$ & 2,25 & 169 & $-7.1 \mathrm{E}-4 \cdot \mathrm{mm}^{2}$ & 0.00159 \\
\hline $\mathrm{u}(\mathrm{A})$ & $\mathrm{N}$ & $\begin{array}{c}30\left(\mathrm{~nm}^{2}\right) \\
0.006\end{array}$ & 3849 & 1 & 0.00003 \\
\hline \multirow[t]{2}{*}{$u(t)$} & $\mathrm{R}$ & $\begin{array}{c}\left({ }^{\circ} \mathrm{C}\right) \\
0.069\end{array}$ & Infinito & $\begin{array}{l}2.1 \mathrm{E}-6 \cdot \mathrm{mm}^{2} /{ }^{\circ} \mathrm{C} \\
2.19 \mathrm{E}-6 \cdot \mathrm{mm}^{2} /^{\circ}\end{array}$ & $1.21 \mathrm{E}-08$ \\
\hline & $\mathrm{R}$ & $\begin{array}{c}\left({ }^{\circ} \mathrm{C}\right) \\
1 \mathrm{E}-05\end{array}$ & Infinito & C & 1.45E-07 \\
\hline$u(\quad)$ & $\mathrm{R}$ & $\left(1 /{ }^{\circ} \mathrm{C}\right)$ & 50 & $-1.1 \mathrm{E}-2 \cdot \mathrm{mm}^{2} \cdot{ }^{\circ} \mathrm{C}$ & 6.30E-08 \\
\hline$u(\operatorname{Lpx})$ & $\mathrm{N}$ & $10(\mathrm{~nm})$ & Infinito & $96 \cdot \mathrm{mm}$ & 9.6E-04 \\
\hline $\mathrm{u}($ Lpy) & $\mathrm{N}$ & $10(\mathrm{~nm})$ & Infinito & $96 \cdot \mathrm{mm}$ & $9.6 \mathrm{E}-04$ \\
\hline
\end{tabular}

Tabela 1. Contribuições para a incerteza de medição 
Para a melhor compreensão da equação 15 , esta pode ser dividida em duas partes. A primeira é a parte inicial da equação onde são combinadas, quadraticamente, todas as grandezas de influência $u(x i)$, seguidas dos seus respectivos coeficientes de sensibilidade. A segunda parte da equação, onde ocorre o somatório dos dois últimos termos da equação, leva em consideração a correlação entre quatro grandezas dividas em dois.

A correlação do modelo de calibração de áreas de aberturas circulares ocorre entre a determinação da borda e a determinação do centro da circunferência. À medida que o programa detecta de forma incorreta um ponto na borda da abertura, o centro da abertura também será determinado de forma incorreta, uma vez que este centro é encontrado através das equações 3 e 4. Essa correlação é considerada duas vezes, uma para cada eixo.

O cálculo da correlação entre as duas grandezas foi feito utilizando as equações 16 e 17, onde as variáreis $\delta_{C x}, \delta_{C y}, \delta_{x i}$ e $\delta_{y i}$ são obtidas provocando estímulos $\delta_{x i}$ que causam alterações no resultado da variável $C_{x}$. A mesma coisa ocorre para as variáveis $\delta_{y i}$ e $C_{y}$.

$$
\begin{aligned}
& r\left(x_{i}, C x\right) \approx \frac{u\left(x_{i}\right) \cdot \delta_{C x}}{u(C x) \cdot \delta_{x_{i}}}=1,33 E-09 \\
& r\left(x_{i}, C x\right) \approx \frac{u\left(y_{i}\right) \cdot \delta_{C y}}{u(C y) \cdot \delta_{y_{i}}}=1,38 E-09
\end{aligned}
$$

O valor encontrado no cálculo da incerteza combinada foi de $0,0015 \mathrm{~mm}^{2}$.

\section{DETERMINAÇÃO DA INCERTEZA EXPANDIDA DE MEDIÇÃO}

Para a determinação da incerteza expandida de medição foi utilizada a incerteza padrão combinada, calculada de acordo com a equação 15 , e o fator de abrangência $k$. Para a determinação deste fator de abrangência foi calculado o grau de liberdade efetivo. (Eq 18).

$$
v_{\text {eff }}=\frac{u_{c}^{4}}{\frac{u^{4}(\bar{A})}{3849}+\frac{u^{4}(C x)}{169}+\frac{u^{4}(C y)}{169}+\frac{u^{4}(\beta)}{50}}=624
$$

O valor obtido no calculo dos graus de liberdade efetivo, de acordo com a tabela para a distribuição-t, retorna como fator de abrangência para uma probabilidade de $95,45 \%$ de confiança, o valor de $k$ igual a 2 . Tornando possível a determinação da incerteza expandida de medição (Eq 19).

$$
U_{95,45 \%}=0,0030 \mathrm{~mm}^{2}
$$


Com o método proposto, o laboratório de metrologia dimensional do Inmetro está apto a atender as demandas relativas a calibração de áreas de aberturas circulares com a exatidão adequada e a incerteza expandida compatível com as requeridas nas medições radiométricas e fotométricas realizadas no Inmetro. Portanto a implantação desta metodologia garante a rastreabilidade nacional para este tipo de padrão e conseqüentemente, para as medições radiométricas e fotométricas.

O modelo matemático apresentado é mais simples que os modelos tradicionais que utilizam o método de mínimos quadrados, o que facilita a determinação das incerteza de medição.

Outra vantagem desta metodologia proposta é a obtenção de um valor final em medida de área para abertura, sem a necessidade de um cálculo posterior para essa determinação.

Em outros sistemas, como apresentado em [6], os resultados finais da medição são obtidos como um raio médio para a abertura e posteriormente a área é calculada.

A partir da analise do modelo matemático, a componente relativa ao tamanho do pixel é a de maior contribuição quando o diâmetro da abertura é aumentado, ou seja, a incerteza de medição é dependente do diâmetro do padrão a ser medido. Para a medição de menores diâmetros $(<1 \mathrm{~mm})$, a maior contribuição passar a ser a determinação do centro da abertura, porém esta contribuição ainda é bastante pequena.

Com base no que foi descrito a respeito do sistema atual apresenta-se como sugestão para trabalhos futuros, aprimoramentos no modelo e no sistema de forma a reduzir a relação entre a incerteza e o tamanho da abertura, para que seja possível alcançar incertezas menores em aberturas menores que $1 \mathrm{~mm}$, e para tornar possível a medição de aberturas maiores que $4 \mathrm{~mm}$.

\begin{abstract}
The Laboratory of Dimensional Metrology of Inmetro is developing a system for measuring the area of circular apertures used in radiometric and photometric measurements. The system is based on the visualization of the area of the aperture with a microscope and the definition of the coordinates of the edges on the captured image. Once this coordinates are obtained, the area of the aperture is calculated using an average radius. The present work aims to present the developed image processing algorithm, the calculations used for the determination of the area and the uncertainty values associated to this method.
\end{abstract}

Keywords : Circular apertures, image analysis, non-contact measuring methods. 


\section{REFERÊNCIAS}

[1] E. Ikonen, P. Toivanen and A. Lassila, "A new optical method for highaccuracy determination of aperture area”, Metrologia, vol. 35, pp. 369-372, 1998.

[2] J. Fowler and M. Litorja, "Geometric area measurement of circular apertures for radiometry at NIST", Metrologia, vol. 40, pp. S9-S12, 2003.

[3] L. Alves, C. Coelho, F. Reis, P. Costa, "Análise da medição da área de abertura apara a realização da candela", $1^{\circ}$ Congresso internacional de metrologia mecânica, Rio de janeiro, Brazil, Oct. 2008.

[4] A. Rezet, "Analytical resolution of Least-Square applications for the circle in interferometry and radiometry", Metrologia, vol. 35, pp. 143-149, 1998.

[5] ISO -International Organization for Standardization, Guide to the expression of uncertainty in measurement. First edition, corrected and reprinted, Switzerland, 1995.

[6] A. Rezet and J. Bastie, "Uncertainty evaluation in non-contact aperture area measurements”, Metrologia, vol. 43, pp. 361-366, 2006. 